NASA/CR-2005-213833

\title{
Computational Model Tracking Primary Electrons, Secondary Electrons, and Ions in the Discharge Chamber of an Ion Engine
}

Sudhakar Mahalingam and James A. Menart

Wright State University, Dayton, Ohio 
Since its founding, NASA has been dedicated to the advancement of aeronautics and space science. The NASA Scientific and Technical Information (STI) Program Office plays a key part in helping NASA maintain this important role.

The NASA STI Program Office is operated by Langley Research Center, the Lead Center for NASA's scientific and technical information. The NASA STI Program Office provides access to the NASA STI Database, the largest collection of aeronautical and space science STI in the world. The Program Office is also NASA's institutional mechanism for disseminating the results of its research and development activities. These results are published by NASA in the NASA STI Report Series, which includes the following report types:

- TECHNICAL PUBLICATION. Reports of completed research or a major significant phase of research that present the results of NASA programs and include extensive data or theoretical analysis. Includes compilations of significant scientific and technical data and information deemed to be of continuing reference value. NASA's counterpart of peerreviewed formal professional papers but has less stringent limitations on manuscript length and extent of graphic presentations.

- TECHNICAL MEMORANDUM. Scientific and technical findings that are preliminary or of specialized interest, e.g., quick release reports, working papers, and bibliographies that contain minimal annotation. Does not contain extensive analysis.

- CONTRACTOR REPORT. Scientific and technical findings by NASA-sponsored contractors and grantees.
- CONFERENCE PUBLICATION. Collected papers from scientific and technical conferences, symposia, seminars, or other meetings sponsored or cosponsored by NASA.

- SPECIAL PUBLICATION. Scientific, technical, or historical information from NASA programs, projects, and missions, often concerned with subjects having substantial public interest.

- TECHNICAL TRANSLATION. Englishlanguage translations of foreign scientific and technical material pertinent to NASA's mission.

Specialized services that complement the STI Program Office's diverse offerings include creating custom thesauri, building customized databases, organizing and publishing research results ... even providing videos.

For more information about the NASA STI Program Office, see the following:

- Access the NASA STI Program Home Page at http://www.sti.nasa.gov

- E-mail your question via the Internet to help@sti.nasa.gov

- Fax your question to the NASA Access Help Desk at 301-621-0134

- Telephone the NASA Access Help Desk at 301-621-0390

- Write to:

NASA Access Help Desk

NASA Center for AeroSpace Information 7121 Standard Drive

Hanover, MD 21076 
NASA/CR-2005-213833

AIAA-2005-4253

\section{Computational Model Tracking Primary Electrons, Secondary Electrons, and Ions in the Discharge Chamber of an Ion Engine}

Sudhakar Mahalingam and James A. Menart

Wright State University, Dayton, Ohio

Prepared for the

41st Joint Propulsion Conference and Exhibit

cosponsored by the AIAA, ASME, SAE, and ASEE

Tucson, Arizona, July 10-13, 2005

Prepared under Grant NAG3-2907

National Aeronautics and

Space Administration

Glenn Research Center 


\section{Acknowledgments}

The authors would like to thank NASA grant monitors Mike Patterson and Thomas Stueber for financially supporting this work. Also the authors would like to thank John Verboncoeur at University of California, Berkeley for providing insightful discussions about XOOPIC and PIC plasma simulations.

Trade names or manufacturers' names are used in this report for identification only. This usage does not constitute an official endorsement, either expressed or implied, by the National Aeronautics and Space Administration.

Available from

NASA Center for Aerospace Information 7121 Standard Drive

Hanover, MD 21076
National Technical Information Service 5285 Port Royal Road Springfield, VA 22100 


\title{
Computational Model Tracking Primary Electrons, Secondary Electrons, and Ions in the Discharge Chamber of an Ion Engine
}

\author{
Sudhakar Mahalingam and James A. Menart \\ Wright State University \\ Dayton, Ohio 45435
}

Computational modeling of the plasma located in the discharge chamber of an ion engine is an important activity so that the development and design of the next generation of ion engines may be enhanced. In this work a computational tool called XOOPIC is used to model the primary electrons, secondary electrons, and ions inside the discharge chamber. The details of this computational tool are discussed in this paper. Preliminary results from XOOPIC are presented. The results presented include particle number density distributions for the primary electrons, the secondary electrons, and the ions. In addition the total number of a particular particle in the discharge chamber as a function of time, electric potential maps and magnetic field maps are presented. A primary electron number density plot from PRIMA is given in this paper so that the results of XOOPIC can be compared to it. PRIMA is a computer code that the present investigators have used in much of their previous work that provides results that compare well to experimental results. PRIMA only models the primary electrons in the discharge chamber. Modeling ions and secondary electrons, as well as the primary electrons, will greatly increase our ability to predict different characteristics of the plasma discharge used in an ion engine.

\section{Nomenclature}

$\begin{array}{llll}A_{g} & \text { Grid surface area } & A_{\theta} & \text { Magnetic vector potential } \\ \vec{B} & \text { Magnetic flux density } & z & \text { Axial position or direction } \\ c & \text { Speed of light } & \Delta r & \text { Grid spacing in radial direction } \\ \vec{D} & \text { Electric displacement } & \Delta t & \text { Time step size } \\ \vec{E} & \text { Electric field } & \Delta z & \text { Grid spacing in axial direction } \\ |e| & \text { Absolute electron charge } & \varepsilon & \text { Kinetic energy of a particle } \\ f_{B} & \text { Beam ion fraction } & \varepsilon_{0} & \text { Permittivity of free space } \\ \vec{H} & \text { Magnetic field strength } & \gamma & \text { Relativistic factor } \\ I & \text { Current } & \lambda D & \text { Debye length } \\ \vec{J} & \text { Current density } & \mu & \text { Permeability } \\ k_{B} & \text { Boltzmann's constant } & v & \text { Collision frequency } \\ \vec{l}, \vec{x} & \text { Position } & \varpi_{p e} & \text { Plasma electron frequency } \\ m & \text { Mass of a particle } & \phi & \text { Electric potential } \\ \dot{m} & \text { Neutral mass flow rate } & \phi_{n} & \text { Grid transparency to neutral particles } \\ N & \text { Number of particles } & \rho & \text { Charge density } \\ \vec{n} & \text { Unit normal } & \sigma & \text { Collision cross-section area } \\ n & \text { Particle number density } & & \end{array}$




$\begin{array}{ll}r & \text { Radial position or direction } \\ S & \text { Surface area } \\ T & \text { Temperature of a particle } \\ \vec{t}, \vec{s} & \text { Rotational vectors } \\ t & \text { Time } \\ \vec{u} & \text { Particle relativistic velocity } \\ V_{c_{-} t i p} & \text { Volume of a cathode tip } \\ V_{d} & \text { Discharge voltage } \\ |\vec{v}| & \text { Particle speed } \\ \vec{v} & \text { Velocity }\end{array}$

$\begin{array}{ll}i & \text { Referring to an } \mathrm{i}^{\text {th }} \text { type charge particle } \\ \text { ion } & \text { Referring to an ion } \\ \text { inc } & \text { Referring to an incident particle } \\ \max & \text { Maximum value } \\ n & \text { Referring to a neutral particle } \\ p e & \text { Referring to a primary electron } \\ r & \text { Referring to radial position or direction } \\ T & \text { Total value } \\ w e i g h t & \text { Particle weighting } \\ z & \text { Referring to axial position or direction } \\ \theta & \text { Referring to azimuthal position or direction }\end{array}$

\section{Introduction}

Ion engines are one of the most promising spacecraft propulsion devices for future missions to distant planets and other celestial bodies. Ion engines have proved themselves to be a successful propulsion system on Deep Space 1. To enhance the development and design of future ion engines it is necessary to improve upon the computational tools available for modeling the performance of an ion engine. The work being presented here is a step in this direction. In this paper a computer code called XOOPIC (ref. 1) is utilized to model the details of the plasma located in the discharge chamber of an ion engine. XOOPIC is a particle-in-cell code developed by the Plasma Theory and Simulation Group at UC, Berkeley. While XOOPIC was not developed for use in ion engine modeling it has been used for a number of plasma application (refs. 2 through 6). In this work XOOPIC will be used to model the plasma in the discharge chamber of an ion engine.

To see where the present work fits into the current state of discharge chamber modeling a brief history of the computational tools that were used for modeling the ion engine's discharge chamber is given here. In the early 1990's Arakawa and Ishihara (ref. 7) developed a complete computational model to predict the behavior of an ion engine discharge chamber. Their computational model is inclusive of the models developed by Arakawa and Yamada, (ref. 8) Arakawa and Wilbur, (ref. 9) and Brophy and Wilbur (ref. 10). Brophy and Wilbur developed a theoretical model to study the performance of an ion engine discharge chamber. Arakawa and Yamada developed a computational tool called PRIMA (refs. 8 and 11) to model the primary electron trajectories using Monte Carlo and finite element methods. PRIMA tracks primary electrons throughout the discharge chamber and includes the effects of magnetic fields; however it does not include the effects of electric fields. Arakawa and Wilbur developed tools to model the magnetic field and plasma present inside the ion engine discharge chamber using a diffusion model. This model looks at the ions as a continuum and considers the movement of ions as a diffusion process. It does not appear as if secondary electrons are modeled. The effects of magnetic fields on the ions are accounted for by using two diffusion coefficients, one parallel to the magnetic field and one perpendicular to the magnetic field. Arakawa and Ishihara combined these two models to simulate the complete performance of an ion engine discharge chamber. In addition they also studied ion motions through the grid holes in their model. Mahalingam and Menart (ref. 11) improved PRIMA and upgraded it to handle any shape of axi-symmetric discharge chamber, increased the computational speed so much finer discretizations could be used, and altered the particle tracking procedure to some extent. Though these models are impressive, there is still a great deal of room for improvement. These models make many assumptions like ignoring electric fields and taking all of the primary electrons to be at the same energy. This is done to make the computer models manageable. The actual physical phenomena occurring inside a discharge chamber is extremely complicated and the need for assumptions will continue to exist. The goal of this work is to develop a model that is more realistic than those discussed above. The way to do this is to use a particle-in-cell technique for the primary electrons, secondary electrons and ions where all these particles are allowed to interact and electric fields are calculated based on the charged particle locations. This is what is done in this work. It should be noted that Hirakawa and Arakawa (ref. 12) developed a computational model that includes electric field effects. However, their model lacks particle collisions, assumes constant particle energy and the study is limited to the cusp region. Recently Wirz and Katz (ref. 13) at JPL developed a 2-D computational tool to model the nonlinear behavior of plasma inside the discharge chamber. Their model includes electric field effects, neutral particles, primary electrons, secondary electrons and positive ions. Currently their computational model seems to be the most advanced computational tool available to model the ion engine discharge chamber. Stueber (ref. 14) at NASA Glenn is developing a 3-D computational model to track the primary electron motions inside the discharge chamber. It is the 
first attempt of a computational tool to include three dimensional effects in the modeling work of an ion engine discharge chamber. Stueber's model only considers primary electrons. The work presented in this paper is the one that includes the physical phenomena: tracking of primary electrons, tracking of secondary electrons, tracking of ions, interaction of charged particles with neutral particles, electric field effects on particles and the particle's affect on the electric fields. In this work the neutral particles are viewed as a uniform background gas through which the charged particles move.

This paper first presents the models used to determine the static fields in the discharge chamber, both magnetic and electric. The magnetic static field is produced by the permanent magnets and the static electric fields are produced by potentials applied to walls of the discharge chamber and the cathode. While the static electric fields are generated within XOOPIC the static magnetic field results are generated external to XOOPIC. Static magnetic field results are passed as an input to XOOPIC. After the static field modeling is presented the mathematical model and computational techniques used by XOOPIC to compute what we will call the dynamic field effects and to compute the trajectories of the particles are presented. The dynamic electric field as well as the dynamic magnetic field modeling is presented in this section because these quantities depend on the location and velocity of the charged particles; unlike the static electric and magnetic fields which do not depend on the charged particle attributes. One of the reasons for presenting the modeling sections in this paper are to give the reader an idea of the detail included in this modeling work. As a test case Hiatt and Wilbur's (ref. 15) $9.2 \mathrm{~cm}$ diameter discharge chamber is modeled with the computational tool presented in this work. While we are not capable of presenting a comparison to Hiatt's and Wilbur's experimentally determined plasma ion production cost, it is the intent of the authors to be able to do this in the near future. Many different types of results for this $9.2 \mathrm{~cm}$ diameter discharge chamber are given in the Results section. Also primary electron number density results obtained with PRIMA are given so that the present results can be compared to them.

\section{Static Field Models}

\section{A. Electromagnetic Fields}

The steady state magnetic field produced by the permanent magnets located on the discharge chamber walls can be modeled using a subset of Maxwell's equations. The governing equation for a 2-D, axisymmetric magnetic field configuration is determined by looking at the magnetostatic part of Maxwell's equations. This magnetic field can be determined with

$$
\frac{\partial}{\partial r}\left(\frac{1}{r \mu} \frac{\partial\left(r A_{\theta}\right)}{\partial r}\right)+\frac{\partial}{\partial z}\left(\frac{1}{\mu} \frac{\partial A_{\theta}}{\partial z}\right)=\frac{\partial H_{c r}}{\partial z}-\frac{\partial H_{c z}}{\partial r}
$$

For the axisymmetric case of a 2-D model, the magnetic vector potential, $A_{\theta}$, only has a circumferential component. The axial and radial magnetic fields are related to the circumferential component of the magnetic vector potential as

$$
B_{r}=\frac{1}{r} \frac{\partial\left(r A_{\theta}\right)}{\partial r}
$$

and

$$
B_{z}=\frac{\partial A_{\theta}}{\partial z}
$$

Equation (1) is solved using following boundary conditions;

$$
\begin{gathered}
A_{\theta} \rightarrow 0 \quad \text { as } \quad r \rightarrow \infty, \\
A_{\theta} \rightarrow 0 \text { as } z \rightarrow-\infty,
\end{gathered}
$$




$$
A_{\theta} \rightarrow 0 \quad \text { as } \quad z \rightarrow \infty
$$

and

$$
\frac{\partial A_{\theta}}{\partial r}=0 \quad \text { at } \quad r=0 .
$$

To utilize these boundary conditions the static magnetic field computational domain must be made larger than the discharge chamber. This is done in this work.

\section{B. Electric Fields}

To determine the electrical potential caused by static electrical potentials, $\phi$ applied to boundaries Laplace's equation (ref. 16) is used

$$
\nabla^{2} \phi=0
$$

The electric field produced by these static potentials is obtained from

$$
\vec{E}=-\nabla \phi
$$

The discharge chamber wall boundary conditions on the electric potentials are given by,

$$
\begin{aligned}
& \phi_{\text {walls }}=50 \text { volts }, \\
& \phi_{\text {cathode }}=0 \text { volts }, \\
& \phi_{\text {screen }}=0 \text { volts } .
\end{aligned}
$$

\section{Particle Models}

\section{A. Dynamic Fields}

For rapidly changing currents and rapidly changing charge densities a more sophisticated model of the electric and magnetic fields caused by the particles in the discharge chamber needs to be considered. This particular model will be called the dynamic electrical and magnetic field model. The dynamic electric and magnetic fields are modeled using the complete set of Maxwell's equations. The integral form of Maxwell's equations is written as, (ref. 16)

$$
\int_{S} \vec{E} \cdot d S=\int \rho d V,
$$




$$
\begin{gathered}
\int_{S} \vec{B} \cdot d S=0, \\
\oint \vec{E} \cdot d \vec{l}=-\int \partial_{t} \vec{B} \cdot d S, \\
\oint \vec{H} \cdot d \vec{l}=\int \partial_{t} \vec{D} \cdot d S+\int \vec{J} \cdot d S .
\end{gathered}
$$

where $\vec{E}$ is the electric field, $\vec{B}$ is the magnetic field, $\vec{H}$ is the magnetic field strength, $\vec{D}$ is the electric displacement, $\rho$ is the charge density, $\vec{J}$ is the current density, $V$ is the volume, $S$ is the surface area, $\vec{l}$ is the position vector and $t$ is the time. Because of the principle of superposition for both the electric and magnetic fields the portion of the fields caused by static phenomena may be added to that caused by the dynamic phenomenon. The total electric and magnetic field at a given point in the discharge chamber is then obtained by adding the two effects. Utilizing this type of technique provides possibilities for speeding up the calculation.

\section{B. Particle Advance}

The governing equations for the particle motion are given by the classical Newton-Lorentz equations of motion (ref. 17). The charge particle equations of motion in the electric and magnetic fields are:

$$
m_{i} \frac{d \vec{v}_{i}}{d t}=q_{i}\left[\vec{E}+\vec{v}_{i} \times \vec{B}\right]
$$

and

$$
\frac{d \vec{x}_{i}}{d t}=\vec{v}_{i}
$$

where $m_{i}$ is the mass of a particle, $\vec{v}_{i}$ is the particle velocity, $q_{i}$ is the electric charge of the particle and $\vec{x}_{i}$ is the particle position. The particle's velocity and position are advanced along a trajectory from the point of its creation until the point it is lost by absorption to a boundary. In this work the electrons inside the discharge chamber are grouped in to two categories. They are: 1) primary electrons and 2) secondary electrons. Primary electrons are the electrons that are emitted from the cathode and they generally have higher kinetic energy than the secondary electrons. Secondary electrons are the electrons produced from the ionization collisions of electrons with the neutral atoms. Essentially both electrons are the same species but they are grouped separately in the analysis. The primary electrons are more important for the discharge chamber performance since they have higher kinetic energy and have more chances of producing ions than the secondary electrons. Modeling them as two groups is useful for presentation purposes.

The boundary conditions for the particles are the particle emission, reflection and absorption. In a general ion engine discharge chamber the primary electrons are produced from a hollow cathode. The plasma near the cathode surface is quasi-neutral (ref. 18). At the exit of the hollow cathode orifice both primary electrons and ions are present. The ions are produced near the cathode tip mainly because of ionizing collisions of primary electrons with the neutrals. This ion production at the cathode tip facilitates a high electron emission current (ref. 19). In this model a plasma source boundary condition is used for the cathode emission. The rate of plasma, $\dot{n}_{P}$, produced is based on the emission current from the cathode, $I_{E}$, and the volume near the cathode tip, $V_{c_{-}}$tip , chosen for plasma generation. It is given by 


$$
\dot{n}_{P}=\frac{I_{E}}{|e| V_{c_{-}} \text {tip }} .
$$

where $|e|$ is the absolute value of the charge of an electron. Both primary electrons and ions are produced at this rate at the cathode tip. The speed at which the primary electrons and ions are leaving the cathode tip is based on the discharge voltage, $V_{d}$, applied and the cathode tip temperature respectively. The speed of the primary electrons, $\left|\vec{v}_{p e}\right|$, leaving the cathode is determined using, (ref. 20)

$$
\left|\vec{v}_{p e}\right|=\sqrt{\frac{3|e| V_{d}}{m_{e}}}
$$

where $m_{e}$ is the mass of an electron and $V_{d}$ is the discharge voltage in $\mathrm{eV}$. Similarly the speed of the ions, $\left|\vec{v}_{\text {ion }}\right|$ that are leaving the cathode tip is calculated using, (ref. 20)

$$
\left|\vec{v}_{i o n}\right|=\sqrt{\frac{3|e| T_{i o n}}{m_{\text {ion }}}}
$$

where $m_{i o n}$ is the mass of an ion and $T_{i o n}$ is the temperature of the ions at the cathode tip in $\mathrm{eV}$.

The electrons are reflected off of cathode biased walls and are absorbed by anode biased walls. The electrons are reflected specularly (ref. 20) back into the discharge chamber at cathode biased surfaces. The ions are absorbed at the discharge chamber walls and at grid locations. Generally the discharge chamber walls are set as anode biased walls and the screen grids at the downstream end of the discharge chamber act as cathode biased walls. All particles are specularly reflected back into the discharge chamber at the axis of symmetry boundary (ref. 1).

\section{Particle Collisions}

Particle collisions modeled for the electrons and ions are collisions with the neutral particles. Coulomb collisions such as electron-electron or electron-ion collisions are not specifically modeled in XOOPIC. In general, a PIC technique models the long range coulomb collisions and smoothes the short range coulomb collision effects (ref. 21). These collisions are modeled inherently in a PIC technique since the charge density at a given location is estimated based on the particle charges that are present within a grid cell volume. This contributes to the long range coulomb collisions, but the collisions of charged particles within a cell volume are underrepresented. Recombination of charge particles is not modeled in this work.

The types of electron collisions with the neutral particles included in this model are elastic, excitation, and ionizing collisions. In an elastic collision, the electron loses little or none of its kinetic energy and gets scattered after the collision. In an elastic collision the electron can pass some of its energy of motion to the motion of the particle with which it is colliding even though none of the energy goes into increasing the internal energy of the particle. In an excitation collision, the electron loses some kinetic energy and gets scattered after the collision. The electron has to lose at least the excitation threshold energy. The excitation threshold energy is the amount of energy required to excite a neutral atom to the next energy level. In an ionizing collision the electron ionizes the neutral atoms and creates a secondary electron. The electron which caused the neutral to be ionized loses kinetic energy equal to or greater than the amount of energy required to ionize the neutral atom. This energy is known as the ionization threshold energy. Ion collisions with neutral particles are modeled as elastic or charge exchange collision types. In an elastic collision, the incident ion loses some or none of its energy and gets scattered. In a charge exchange collision, the incident ion excites a colliding neutral particle and after the collision the neutral particle becomes an ion and the incident ion becomes a neutral particle. 
The occurrence of a particle collision is determined using a null collision technique (refs. 22 and 23). This technique uses a constant collision frequency to determine the maximum fraction of particles that undergo collisions. This method eliminates the look up of kinetic energy for each particle. The null collision probability (ref. 23) is given by,

$$
P_{\text {null }}=1-\exp \left(-v_{c} * \Delta t\right)
$$

where $v_{c}$ is the constant collision frequency obtained by looking for the maximum sum of all collisions over all possible energies.

$$
v_{c}=\max \left(n_{n} \sigma_{T}\left(\varepsilon_{i n c}\right)\left|\vec{v}_{i n c}\right|\right) .
$$

This is a computation convenience that is made to represent reality by adding a null collision frequency to the actual collision frequency at each energy level (see reference [23]). Here the neutral number density, $n_{n}$ is given by (ref. 10)

$$
n_{n}=\left[4 \dot{m}\left(1-\eta_{u}\right)\right] /\left|e \| \vec{v}_{n}\right| A_{g} \phi_{n},
$$

where $A_{g}$ is the grid area, $\dot{m}$ is the propellant mass flow rate, $\eta_{u}$ is the propellant utilization parameter, $\left|\vec{v}_{n}\right|$ is the velocity of the neural particles, and $\phi_{n}$ is the grid transparency to neutral atoms. The total collision cross-section, $\sigma_{T}\left(\varepsilon_{i n c}\right)$, is given by

$$
\sigma_{T}\left(\varepsilon_{i n c}\right)=\sigma_{1}\left(\varepsilon_{i n c}\right)+\sigma_{2}\left(\varepsilon_{i n c}\right)+\ldots+\sigma_{N}\left(\varepsilon_{i n c}\right)
$$

where $N$ is the total number of collision types considered. The collision cross section area for all collision types are based on the kinetic energy of the incident particle which is given by,

$$
\varepsilon_{i n c}=\frac{1}{2} m_{i n c}\left|\vec{v}_{i n c}\right|^{2}
$$

where $m_{i n c}$ is the mass of the incident particle and $\left|\vec{v}_{i n c}\right|$ is the speed of the incident particle. Since uniform neutral number density is assumed the constant collision frequency becomes,

$$
v_{c}=n_{n} * \max \left(\sigma_{T}\left(\varepsilon_{i n c}\right)\left|\vec{v}_{i n c}\right|\right) \text {. }
$$

The null collision probability is estimated using equation (22) with equation (27). The number fraction of particles that experience collisions is calculated based on this null collision probability,

$$
N_{\text {coll }}=N_{\text {total }} * P_{\text {null }} \text {. }
$$

Only this number of particles is tested for collisions and some of these particles may not undergo a collision. 


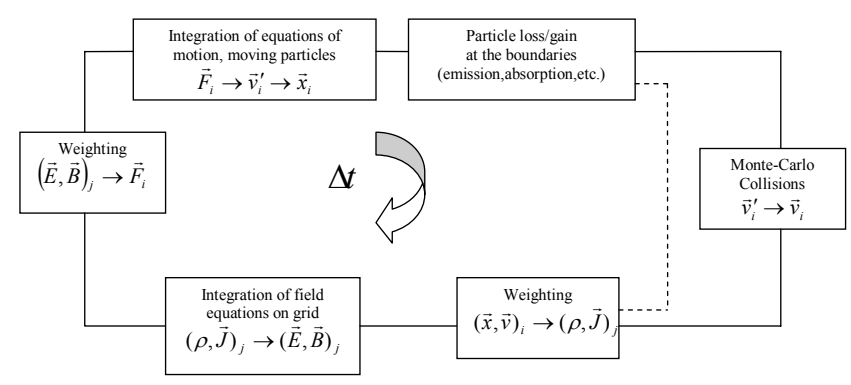

Figure 1.-A Flow chart of PIC-MCC model shows computing sequence of a plasma simulation problem

(from Vahedi and Surendra, 1995).

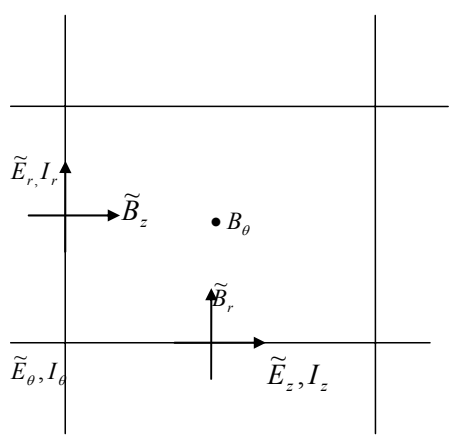

Figure 2.-Field variables are stored as used in the Yee mesh.

\section{Solution of Mathematical Model}

In this section the solution of the mathematical models presented above is discussed. Of course numerical techniques are utilized to solve these equations. A general PIC-MCC (ref. 21) flow chart (see fig. 1) shows the computing sequence of how XOOPIC models a plasma simulation problem for each time step, $\Delta t$. In the following sections the details of how the field and particle calculations are advanced through time are given. Details on how the collisions fit into this process are also given.

\section{A. Electromagnetic Fields}

The field effects of any plasma device in XOOPIC are modeled on a two dimensional computational mesh. It handles orthogonal meshes of $x-y$ coordinates and $r-z$ coordinates. In our model we use cylindrical $r-z$ coordinates since most of the ion engine discharge chamber configurations studied are cylindrically shaped. In XOOPIC the field variables, as shown in figure 2, are stored at the cell centers which are followed in the fashion as used on the Yee mesh (ref. 24).

The electric and magnetic field problems are solved by utilizing two modes: static mode and dynamic mode. The static mode is solved once at the beginning of the program and utilized throughout the computation. The dynamic quantities are solved for each time step because they depend on the position of the particles. These two portions of the field solver can be handled separately because of the principle of superposition.

The static magnetic fields are solved utilizing the computer code called MAXWELL-2D (ref. 25). MAXWELL$2 \mathrm{D}$ is a computer program developed by the Ansoft Corporation, Inc. A previous study by Menart (ref. 26) indicates that Maxwell-2D is an excellent software package for modeling two dimensional magnetic field problems. The details of the discharge chamber configuration, such as the location and orientation of the magnets and the magnet properties, are required inputs to Maxwell-2D. If the wall material interacts with the magnetic field their location and magnetic properties are also required. Maxwell-2D uses a finite element technique with an iterative or direct matrix solver to find the magnetic vector potential values. The magnetic field values are obtained using equations (2) and (3) which are then provided as an input to XOOPIC.

The static electric fields are determined inside XOOPIC itself. XOOPIC has the option of choosing different types of Laplace equation solvers for any given problem. The Laplace solvers available in XOOPIC are: multigrid method, conjugate gradient algorithm, dynamic ADI algorithm, and generalized minimum residual algorithm. Any one of these algorithms can be used to solve Laplace's equation. The potential values are obtained at the cell nodes. Then the corresponding $E$ field values are calculated from equation (9).

The dynamic portion of this field analysis is the most difficult. As can be seen in equations (15) and (16) the electric fields produced by time varying magnetic fields and the magnetic fields produced by time varying electric fields are included in the analysis. For the discharge chambers that are studied in this work neither one of these phenomena is very important. The currents produced in a discharge chamber are too small to produce any significant magnetic fields and charge densities do not change fast enough to cause electrical fields changes to produce noticeable magnetic fields. The important part of this dynamic analysis for ion engines is tracking the change in the electric fields caused by the changing particle locations. This is effectively done in XOOPIC. A set of magnetic field and electric field equations is obtained by using a time centered finite differencing on equations (15) and (16). Leapfrog scheme is adopted for the time integration of these field equations. The details of advancing electromagnetic field problems are discussed at length in reference 1. 


\section{B. Particle advance}

XOOPIC uses the relativistic form of the equation of motion for charged particles in electric and magnetic fields. Time integration of these equations are handled using a Boris advance technique (ref. 17). The time centered finite difference form of the relativistic equation of motion is given as,

$$
\frac{\vec{u}_{i}^{n+1 / 2}-\vec{u}_{i}^{n-1 / 2}}{\Delta t}=\frac{q_{i}}{m_{i}}\left[\vec{E}^{n}+\frac{\vec{u}_{i}^{n+1 / 2}+\vec{u}_{i}{ }^{n-1 / 2}}{2 \gamma_{i}{ }^{n}} \times \vec{B}^{n}\right]
$$

where $\vec{u}_{i}$ is the particle relativistic velocity which is equivalent to $\vec{u}_{i} \equiv \gamma_{i} \vec{v}_{i}$. The relativistic factor, $\gamma_{i}$, is calculated using the relation, $\gamma_{i}^{2}=1+\frac{\left|\vec{u}_{i}\right|^{2}}{c^{2}}$, where $c$ is the speed of light. In the Boris advance the electric and magnetic forces are separated in the integration by substituting the relations

$$
\begin{aligned}
& \vec{u}_{i}^{n-1 / 2}=\vec{u}_{i}^{-}-\frac{q_{i} \vec{E}^{n} \Delta t}{2 m_{i}} \\
& \vec{u}_{i}^{n+1 / 2}=\vec{u}_{i}^{+}+\frac{q_{i} \vec{E}^{n} \Delta t}{2 m_{i}}
\end{aligned}
$$

into equation (29) giving a new time integration equation which eliminates the electric field forces from the finite difference equation,

$$
\frac{\vec{u}_{i}^{+}-\vec{u}_{i}^{-}}{\Delta t}=\frac{q_{i}}{2 \gamma_{i}{ }^{n} m_{i}}\left(\vec{u}_{i}^{+}+\vec{u}_{i}^{-}\right) \times \vec{B}^{n} .
$$

Equation (32) represents the rotation caused by magnetic field forces on the charged particles. The magnetic field rotation is handled by a Boris rotation which introduces another set of variables,

$$
\vec{u}_{i}^{\prime}=\vec{u}_{i}^{-}+\vec{u}_{i}^{-} \times \vec{t}_{i}
$$

and

$$
\vec{u}_{i}^{+}=\vec{u}_{i}^{-}+\vec{u}_{i}^{\prime} \times \vec{s}_{i}
$$

with

$$
\vec{t}_{i}=\frac{q_{i} B^{n} \Delta t}{2 \gamma_{i}{ }^{n} m_{i}}
$$


The computational steps are: first compute the $\vec{u}_{i}^{-}$velocity by adding half the electrical force to the known velocity, $\vec{u}_{i}{ }^{n-1 / 2}$, at time level $n-1 / 2$ using equation (30). Then rotate the $\vec{u}_{i}^{-}$velocity using equations (33) to (36) for a full time step to calculate $\vec{u}_{i}{ }^{+}$velocity and add the remaining half electrical impulse, equation (31), to obtain the new velocity, $\vec{u}_{i}^{n+1 / 2}$ at time level $n+1 / 2$. The particle positions are updated with new velocity,

$$
\vec{x}_{i}^{n+1}=\vec{x}_{i}{ }^{n}+\frac{\vec{u}_{i}{ }^{n+1 / 2} \Delta t}{\gamma_{i}^{n+1 / 2}}
$$

where $\gamma_{i}^{n+1 / 2}$ is evaluated at time level $n+1 / 2$.

In an actual hollow cathode, the particles emitted from the cathode tip area, are from any arbitrary location within this area and with a random velocity. This random emission of primary electrons and ions from the cathode is handled using a Markov-Chain Monte Carlo scheme. The number of particles that are emitted for a given time step is determined with the source rate, cathode tip volume as given in equation (19). The speed of each primary electron and ion are calculated using equations (20) and (21) which utilizes the isotropic temperature values given as input. A Maxwellian velocity distribution is applied in each direction to get a velocity for each particle. For the primary electrons their temperature is equivalent to the discharge voltage of cathode. For the ions their temperature would be the temperature near the hollow cathode tip.

\section{Particle collisions}

The neutral particles are assumed distributed uniformly throughout the discharge chamber. The neutral temperature used in this model is $0.025 \mathrm{eV}$ which is equivalent to $290 \mathrm{~K}$. The neutral number density is calculated using equation (24). The maximum value of $\sigma_{T}\left(\varepsilon_{\text {inc }}\right)\left|\vec{v}_{\text {inc }}\right|$ is estimated by calculating the total collision cross section areas, $\sigma_{T}\left(\varepsilon_{i n c}\right)$, and the particle speed, $\left|\vec{v}_{\text {inc }}\right|$, for a range of kinetic energies, $\varepsilon_{\text {inc }}$, from 0 to 1000 electron volts. From this range of particle kinetic energies the maximum value of the collision swept volumetric rate is calculated. Then utilizing the neutral number density and the maximum collision volume rate, the constant collision frequency, $v_{c}$, is obtained using equation (23). The probability of null collision is calculated using a constant collision frequency and time step, $\Delta t$. Then the fraction of particles, $N_{\text {coll }}$ that undergoes particle collisions is estimated. The selection of which particles should have a collision is done through a random process. In this process effort is made not to duplicate the same particle for another type of collision in a given time step. Then the collision type for each colliding particle is tested using a random number, $R$, between 0 and 1 in the following manner,

$$
\begin{array}{cc}
R \leq \mathrm{v}_{1}\left(\varepsilon_{i n c}\right) / \mathrm{v}_{c} & \text { (Collision Type 1) } \\
\mathrm{v}_{1}\left(\varepsilon_{\text {inc }}\right) / \mathrm{v}_{c}<R \leq\left(\mathrm{v}_{1}\left(\varepsilon_{\text {inc }}\right)+\mathrm{v}_{2}\left(\varepsilon_{\text {inc }}\right)\right) / \mathrm{v}_{c} & (\text { Collision Type 2) } \\
\vdots & \\
\sum_{j=1}^{N} \mathrm{v}_{j}\left(\varepsilon_{i n c}\right) / \mathrm{v}_{c}<R & \text { (Null collision) }
\end{array}
$$


This collision process is performed for the primary electrons, the secondary electrons and the ions in the same way. In the case of electron particles three collision types: elastic, excitation and ionization are modeled. For ion particles two collisions types, elastic and charge exchange, are modeled. The collision model used in XOOPIC to handle these particle collisions are from the work of Vahedi and Surendra (ref. 23). More details about collisions can be obtained from their paper (ref. 23). Also XOOPIC uses collision cross-section areas for the elastic and excitation collisions from Surendra et al. (ref. 27) and the ionization collision cross section areas from Rapp and Golden (ref. 28) for the argon gas.

\section{Stability Criteria}

XOOPIC uses an explicit finite difference time domain (FDTD) algorithm for solving the electromagnetic field equations and an explicit leap frog time mover for the particle advancing. These explicit schemes have limitations on selecting the grid spacing sizes, $\Delta z$ and $\Delta r$, and the time step size, $\Delta t$. The FDTD algorithm for the electromagnetic solver needs to meet the Courant condition (ref. 17) to control the nonphysical growth of numerical errors. The Courant condition is

$$
c \Delta t \sqrt{\frac{1}{\Delta z^{2}}+\frac{1}{\Delta r^{2}}} \leq 1,
$$

where $c$ is the speed of light. The explicit particle advancing scheme (ref. 17 and 21) is limited by the following stability constraint on the time step size,

$$
\varpi_{p e} \Delta t \leq 2,
$$

and for accuracy,

$$
\varpi_{p e} \Delta t \approx 0.2,
$$

where $\varpi_{p e}$ is the plasma frequency and it is given by (ref. 29)

$$
\varpi_{p e}=\sqrt{\frac{n_{e}|e|^{2}}{\varepsilon_{0} m_{e}}} .
$$

The constraint for grid spacing is given as, (ref. 21)

$$
\lambda_{D} \sqrt{\frac{1}{\Delta z^{2}}+\frac{1}{\Delta r^{2}}} \geq 0.3,
$$

where $\lambda_{D}$ is the Debye length and it is given by, (ref. 29) 


$$
\lambda_{D}=\sqrt{\frac{\varepsilon_{0} k_{B} T_{e}}{n_{e}|e|^{2}}} .
$$

Here $n_{e}$ is the electron number density and $T_{e}$ is the electron temperature. The constraint for grid spacing is required to control the plasma heating (ref. 17) which happens with larger grid sizes. The particle weighting is also a factor to maintain the stability of the numerical plasma simulations. The numerical noise is proportional to $\sqrt{1 / N_{c p}}$, where $N_{c p}$ is the number of macro particles in the simulation. Weighting each particle with fewer physical particles will reduce the numerical noise. In general too few particles can result in numerical heating.

\section{Test Model}

In this work an experimental discharge chamber design studied by Hiatt and Wilbur (ref. 15) is used as a test model. The reason for choosing this model is because previous modeling studies have used this chamber design for comparisons. Hiatt and Wilbur used a $9.2 \mathrm{~cm}$ diameter steel walled discharge chamber to perform experimental investigations. The chamber length used on this model is $11.8 \mathrm{~cm}$. Figure 3 shows the schematic view of this experimental model. It is a straight cylindrical shaped discharge chamber. It has two samarium cobalt magnets which are placed inside the discharge chamber. Magnet 1 is placed on the back wall and Magnet 2 is placed on the side wall at a distance of $3.7 \mathrm{~cm}$ from the screen grid. The magnetic flux density of the magnets is maintained at 2700

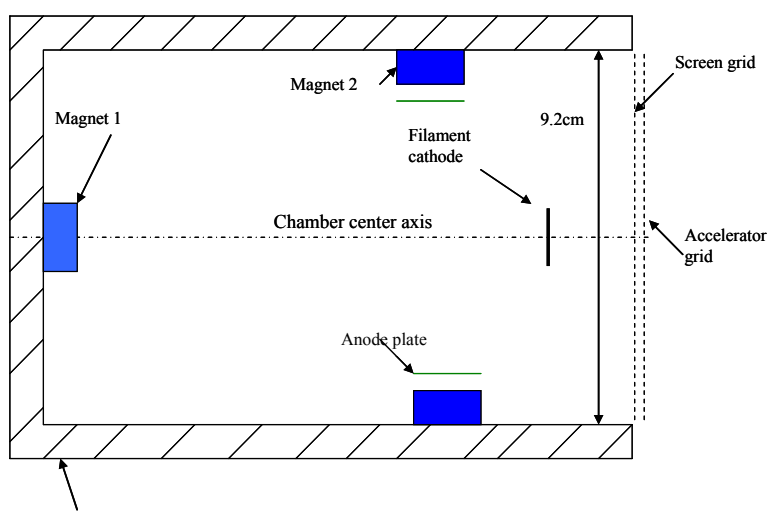

Figure 3.- - Hiatt and Wilbur's experimental model. Gauss. The propellant used in this model is argon. An anode plate is placed axially at the bottom surface of Magnet 2. A circular, filament type cathode of $1 \mathrm{~cm}$ diameter is used in this experimental setup. This cathode is made from two coiled tungsten wires of $0.25 \mathrm{~mm}$ diameter which are wound together to form a spiral hoop which has a diameter of $1 \mathrm{~cm}$. This hoop shape keeps the cathode off the center line. This cathode is located $1.5 \mathrm{~cm}$ upstream of the screen grid surface. The electric potentials at the wall boundaries, anode surface, cathode surface and screen grid surface need to be specified before performing simulations in XOOPIC. From Hiatt and Wilbur's paper it is found that screen grid was maintained at 750 volts and the discharge voltage was 50 volts. Generally in ion engine discharge chambers, the cathode and the screen grid surfaces are maintained at the same potential (ref. 30) and the anode walls are kept at a higher potential to get the cathode discharge voltage. This procedure is followed in this work. The cathode, the discharge chamber walls, and the screen grid are at zero potential and the anode plate is maintained at 50 volts. The neutral number density is found from the relation given by equation (24). This equation gives a neutral number density of $3.3 \times 10$ (ref. 19) $\# / \mathrm{m}^{3}$ for the mass flow used in this paper.

Hiatt and Wilbur performed tests for a range of argon mass flow rates from 100 to $700 \mathrm{~mA}$ eq. They measured the discharge current, $I_{D}$, the ion beam current, $I_{B}$, and the total ion production current, $I_{P}$ for each mass flow rate. These measured currents were used to compute the plasma ion production cost, $\varepsilon_{P}$, and the extracted ion fraction, $f_{B}$ utilizing the relations below (ref. 15):

$$
f_{B}=I_{B} / I_{P},
$$

and

$$
\varepsilon_{P}=\left(I_{D}-I_{P}\right) V_{d} / I_{P} .
$$


Hiatt and Wilbur present $\varepsilon_{P}$ results in a plot against the neutral density parameter, $\dot{m}\left(1-\eta_{u}\right)$, for each mass flow rate. In this work the case of maximum mass flow rate, $648 \mathrm{~mA}$ eq., and the neutral density parameter, $\dot{m}\left(1-\eta_{u}\right)$, of $610 \mathrm{~mA}$ eq is taken. For this test case, the plasma ion production cost, $\varepsilon_{P}$ is found to be $70 \mathrm{eV}$ from their plot. Also the extracted ion fraction, $f_{B}$ is found to be 0.23 from their paper. Also needed are the cathode emission current, $I_{E}$, and the cathode tip volume to calculate the plasma source rate. From Brophy's model, the cathode emission current is given by, (ref. 10)

$$
I_{E}=I_{D}-I_{P}
$$

Substituting $\varepsilon_{P}$ and $f_{B}$ values into equations (45) to (47) gives the emission current. The emission current is found to be $232 \mathrm{~mA}$. The cathode tip volume is determined by assuming the tip as a circular cylindrical ring. It is given by,

$$
V_{c_{-} \text {tip }}=\pi\left(r_{\text {in }}^{2}-r_{\text {out }}^{2}\right) h
$$

where $r_{i n}$ is the inner radius of the cathode hoop, $r_{\text {out }}$ is the outer radius of the cathode hoop, and $h$ is the length of the cathode coil. Since the cathode details from Hiatt and Wilbur's paper were not clear we assume a 1 mm circular coil thickness and a $1 \mathrm{~mm}$ length for the coil. This assumption gives an inner hoop radius of the cathode wire of $5 \mathrm{~mm}$, an outer hoop radius of $6 \mathrm{~mm}$ and wire width of $1 \mathrm{~mm}$. Substituting these values into equation (48) gives the cathode tip volume. Using equation (19) the plasma source rate can be obtained. The plasma source rate used in this work is $3.6 \times 10$ (ref. 25$) \# /\left(\mathrm{m}^{3}-\mathrm{s}\right)$. This parameter is used to get the number of primary electrons and ions emitted from the cathode tip at a given time step.

The numerical parameters chosen to perform this simulation in XOOPIC are: the grid spacing, $\Delta z$ and $\Delta r$, the time step, $\Delta t$, and the particle weighting. These parameters are given in equations (39), (40), (41), and (43). The grid spacing parameters are based on the Debye length and the time step size is based on the plasma frequency. $\lambda_{D}$ and $\varpi_{p e}$ need to be estimated for this discharge chamber model. For the plasma in the discharge chamber of an ion engine a high value of the electron number density (ref. 31) is $1 \times 10^{18} \# / \mathrm{m}^{3}$. This number density value with a range of electron temperatures from 10 to $50 \mathrm{eV}$, is used in equation (44) to obtain a set of Debye length values. The axisymmetric chamber dimensions of $11.8 \mathrm{~cm}$ length and $4.6 \mathrm{~cm}$ radius are used to get the grid spacing values. The grid spacing values and the debye lengths are checked to select a grid spacing that meets the debye length limit. Using 1000 grid points in the axial direction and 500 grid points in the radial direction satisfies the debye length limit for the range of electron temperatures mentioned above. Similarly the plasma frequency is calculated using equation (42) by utilizing the same high electron number density value. It is found to be $5.63 \times 10^{10} \mathrm{~s}^{-1}$. The Courant condition in equation (39) puts a smaller limit on the time step value. For this reason a time step value of $2 \times 10^{-12} \mathrm{~s}$ was used. This value worked well. We can select an even finer grid spacing and a smaller time step to improve the accuracy of these simulations. However, at present time the grid spacing is limited by the availability of computer memory. A smaller time step is not used to keep the computational time from becoming undoable. The particle weighting also plays a role in the accuracy. In this work a particle weighting of $1 \times 10^{6}$ real particles per computational particle is used to maintain the numerical noise small while not making the computations excessively long. The numerical parameters considered used in the computation are:

$$
\begin{gathered}
\Delta z=1.18 \times 10^{-4} \quad m, \\
\Delta r=0.92 \times 10^{-4} \quad m, \\
\Delta t=2 \times 10^{-12} \mathrm{~s} \\
N_{\text {weight }}=1 \times 10^{6} \quad \text { real particles/computational particles . }
\end{gathered}
$$




\section{RESULTS}

In this section the results from Maxwell-2D and XOOPIC for the test model are presented. From Maxwell-2D the magnetic field results are obtained. These results provide information about the magnetic field values at different locations inside the discharge chamber. These values are essentially constant for all times in the discharge chamber and thus they are only shown once.

From XOOPIC numerous results are obtained. The results are presented in two categories: particle and electric field. For the most part these results are given at 2 and $300 \mathrm{~ns}$, except for the electrical potential and the total number of particles in the discharge chamber. The electrical potential is shown at time zero so that the electrical potentials caused by the applied boundary conditions can be seen. Any change in these values is caused by the particles. The total number of particles is shown as a function of time in the discharge chamber. Results from 2 and 300 ns are presented next to each other so that comparisons can be made. The last result presented in this paper is that from PRIMA. This result gives the primary electron number density in the discharge chamber at steady state conditions.

\section{A. Initial Results}

The magnetic vector potential contours are given in figure 4. This plot shows how the magnetic field lines run between the two magnets. A 100 gauss-cm contour line forms near the cathode and ends at the center of the anode plate placed below Magnet-2. This contour line will tend to direct the motion of the primary electrons. Figures 5 and 6 show the contour plots of radial and axial magnetic fields. Strong negative radial field lines are observed below the Magnet-2 surface. The magnetic field strength is decreasing radially as we move downward from the second magnet axial surface. Also looking from figure 5 and figure 6 we can see that the cusp region at the Magnet- 2 surface has stronger radial field components and weaker the axial field components. This indicates that the radial moving electron particles will have an easy path of moving into the cusp region and axially moving electron particles will be confined by the strong radial magnetic field.

The electric potential at time zero is shown in figure 7. This result is obtained from XOOPIC by solving the Laplace equation of electric potential. This plot shows zero potential value at the discharge chamber walls and at the screen grid areas. Also the potential value at the anode plate surface is 50 volts. This 50 volts value is diffusing to zero value around the anode wall surface.

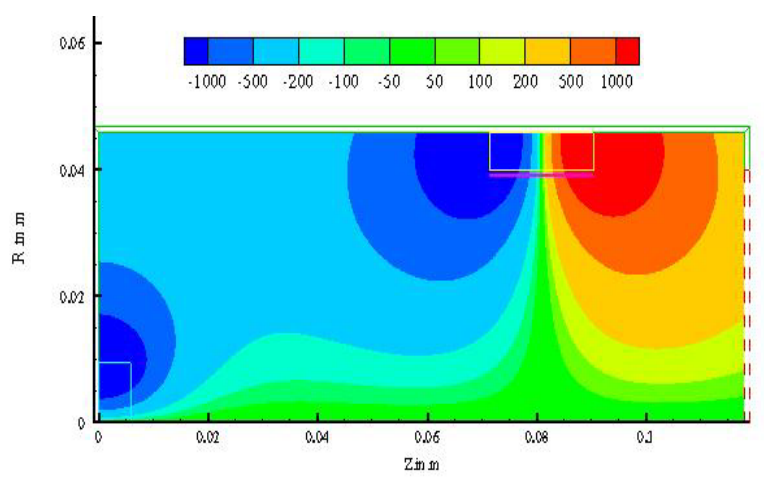

Figure 4.- Magnetic vector potential contour lines in gauss-cm.

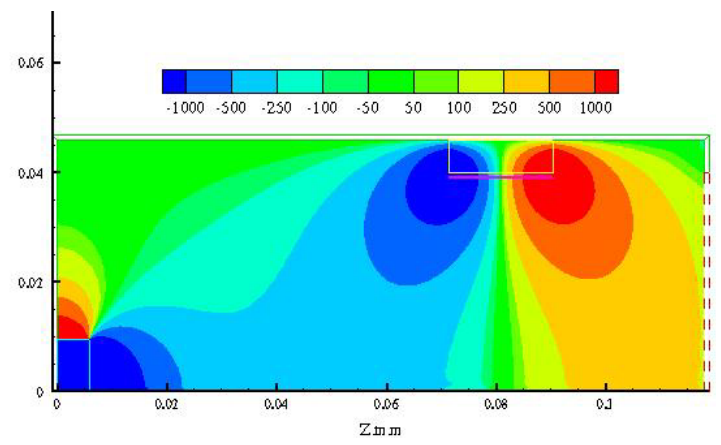

Figure 6.-Axial Magnetic field contour lines in gauss.

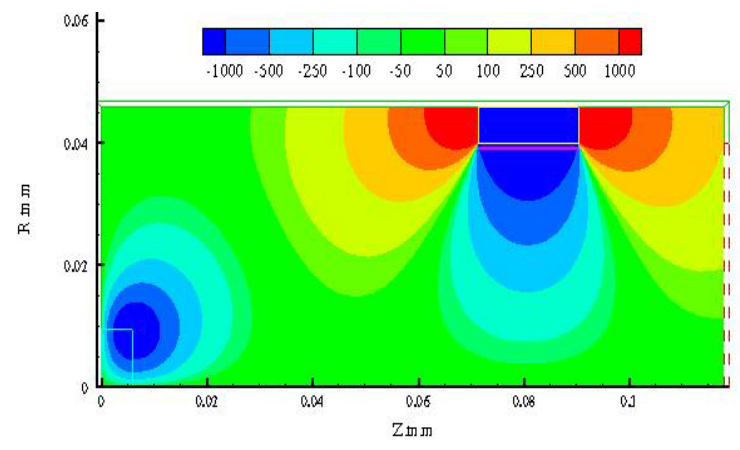

Figure 5.- Radial magnetic field contour lines in gauss.

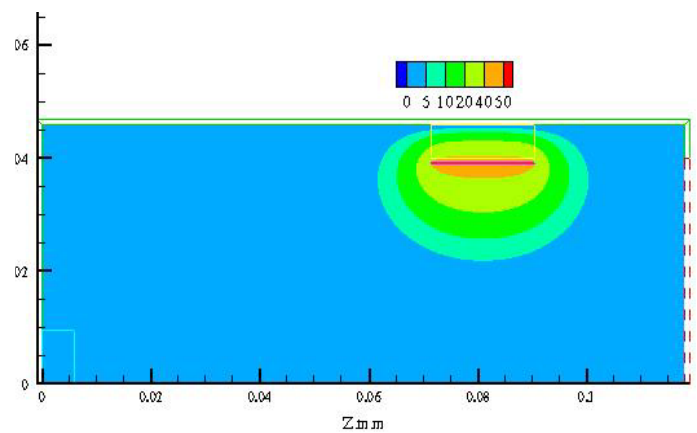

Figure 7.-Electric potential contour lines in volts. 


\section{B. Particle Results}

In this section the results of primary electrons, secondary electrons and ions inside the discharge chamber are presented. A plot of total number of primary electrons, secondary electrons, and ions present inside the discharge chamber is shown in figure 8 . Both the vertical and horizontal axes in this plot have a logarithmic scale. The total number of particles includes all the physical particles present inside the discharge chamber. This

value is obtained by multiplying the number of macro particles with its weighting. This plot indicates that all particles are steadily increasing with time. Primary electrons and argon ions are increasing at the same rate until $40 \mathrm{~ns}$. After $40 \mathrm{~ns}$, the ions are increasing at a slightly higher rate than the primary electrons. This difference in rate of increase for ions and primary electrons is due to the increase in number of ionizing collisions of primary electrons with the neutrals. The first secondary electron is formed at $0.8 \mathrm{~ns}$. By looking at figure 8 , we can say that steady state operation of this discharge chamber is not yet achieved and the simulation still needs to be run for a long time. It took 5 days of computational time on a single processor to compute this simulation up to a time of $300 \mathrm{~ns}$. This is the limit of computational time available for this work. In the future the computer code will be parallelized and steady state will be reached.

The primary electron number density results at 2 and at $300 \mathrm{~ns}$ are presented in figure 9 and figure 10 respectively. By Looking at figure 9 one can see that few primary electrons are present inside the discharge chamber at $2 \mathrm{~ns}$. The ones that are present at 2 ns are located near the cathode. From figure 10, it can be seen that the region of primary electron movement is along the magnetic field lines that run from the cathode to the anode located above the side wall magnet (see fig. 4 and fig. 10). While it is difficult to see in figure 10 some primary electrons are making their way towards the cusp region of the back wall magnet. For the most part the primary electrons are spread near the cathode radially for a height of $1.2 \mathrm{~cm}$. The maximum number density value of $3.3 \times 10^{18} \# / \mathrm{m}^{3}$ is observed above the cathode and the number density value near the screen grid is about $10^{14} \mathrm{\#} / \mathrm{m}^{3}$.

The secondary electron number density results are also interesting to see. These electrons are created due to the ionization collisions of primary electrons with the neutrals. Figure 11 shows the secondary electron number density contours at $300 \mathrm{~ns}$. The results of 2 ns case are not shown here because the number of secondary electrons produced at $2 \mathrm{~ns}$ is very small, around $2 \times 10^{7}$. At $300 \mathrm{~ns}$, a significant number of secondary electrons are present inside the discharge chamber. A high value of secondary electron number density, $1 \times 10^{18}$ $\# / \mathrm{m}^{3}$ is observed near the cathode location. The secondary electron number density decreases rapidly at locations away from cathode. Only a few secondary electrons are present in the cusp region at $300 \mathrm{~ns}$.

The argon ions are produced from the cathode tip and from ionizing collisions of electrons with the argon neutrals. The argon ion number density values inside the discharge chamber

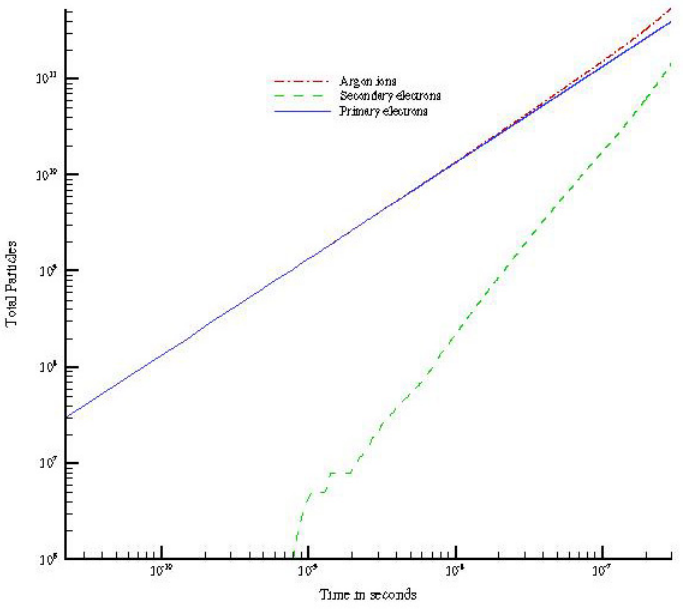

Figure 8.-Total number of particles vs. time.

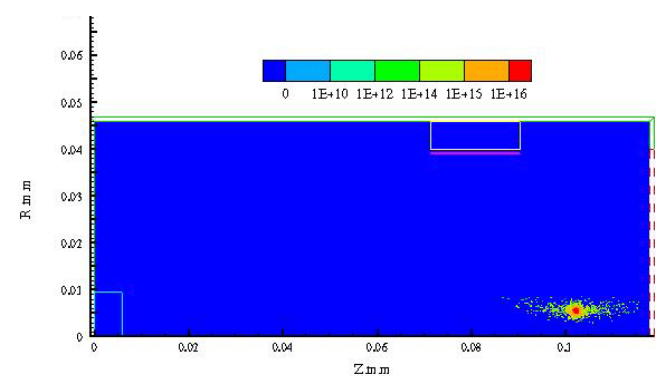

Figure 9.-Primary electron number density contour lines in $\# / \mathrm{m}^{3}$ at $2 \mathrm{~ns}$.

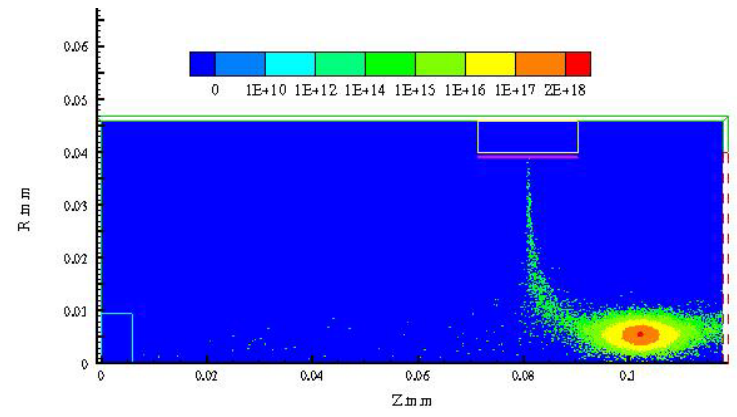

Figure 10.-Primary electron number density contour lines in $\# / \mathrm{m}^{3}$ at $300 \mathrm{~ns}$.

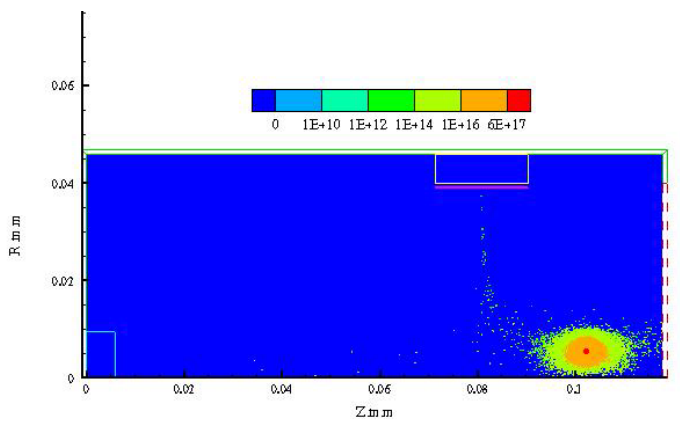

Figure 11.- Secondary electron number density contour lines in $\# / \mathrm{m}^{3}$ at $300 \mathrm{~ns}$. 


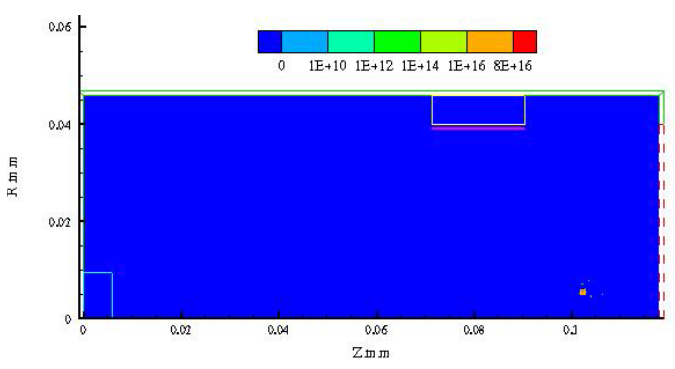

Figure 12.-Argon ion number density contour lines in $\# / \mathrm{m}^{3}$ at $2 \mathrm{~ns}$.

at $2 \mathrm{~ns}$ are given in figure 12 . The maximum number density value is observed at the cathode. The speed of ions is two to three orders of magnitude less than the electron speed because ions are much heavier than electrons. This is the reason the ions have not moved as far as the primary electrons at 2 ns (see fig. 9). Figure 13 shows the argon ion number density values at $300 \mathrm{~ns}$. This plot shows the ions moving away from the cathode region. The interesting result from this density plot is that the ions are moving radially much further into the stronger axial magnetic field region. This stronger axial field significantly confines the electrons, but the ions cross these lines and move further into the strong regions. This movement of ions into the stronger magnetic field region is not surprising because the Larmor radii (ref. 16) for the argon ions are much higher than the Larmor radii for the electrons. A high value of the argon ion number density of $4.5 \times 10^{18} \mathrm{\#} / \mathrm{m}^{3}$ is observed at the cathode.

\section{Field Results}

In this section the results of the electric potential are presented. The electric potential results at 2 and at $300 \mathrm{~ns}$ are shown in figures 14 and 15 . The electric potential values observed near the cathode area are high in both cases. From figure 14, it can be seen that the region of high potential is limited to the cathode tip area because all argon ions are confined in this small region. There is a net positive charge density in this region because the primary electrons move out quicker than the ions. Results at $300 \mathrm{~ns}$ shown in figure 15 indicate a wider area of high electric potential. This is due to the movement of ions away from the cathode tip into the interior of ion engine discharge chamber while the primary electrons continue to move away even further.

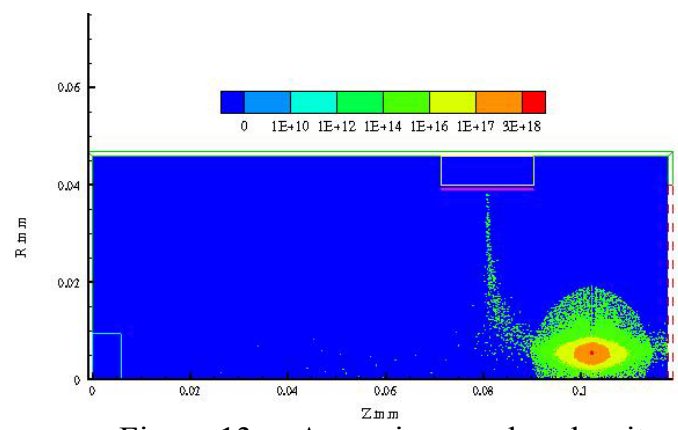

Figure 13.-Argon ion number density contour lines in $\# / \mathrm{m}^{3}$ at $300 \mathrm{~ns}$.

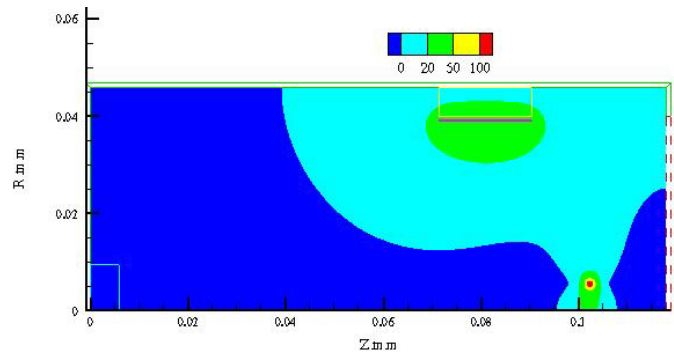

Figure 14.-Electric potential contour lines in volts at 2 ns.

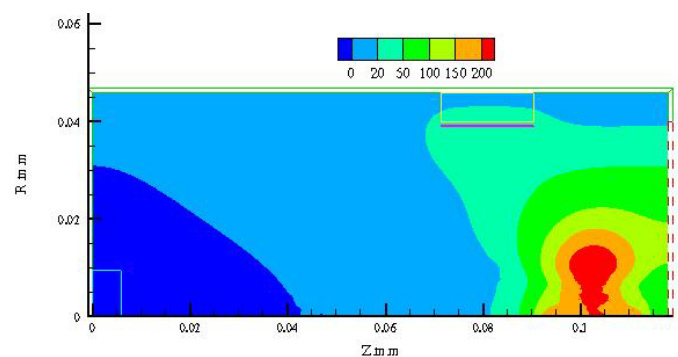

Figure 15.-Electric potential contour lines in volts at $300 \mathrm{~ns}$.

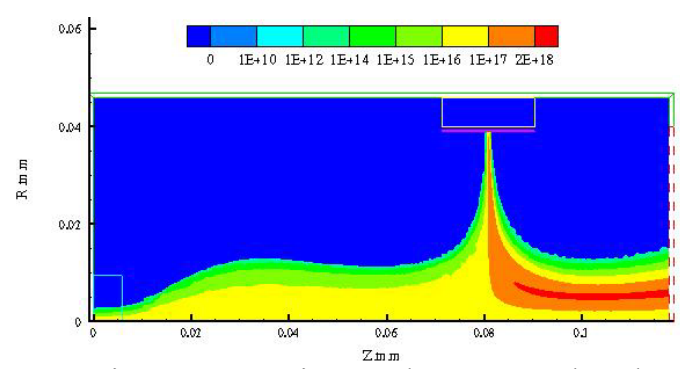

Figure 16.-Primary electron number density results from PRIMA in $\# / \mathrm{m}^{3}$.

\section{Comparisons}

To get a feel for how the computational results from XOOPIC are comparing to results obtained from PRIMA figure 16 is given. The results in figure 16 show the relative primary electron number densities as obtained from PRIMA for the Hiatt and Wilbur's model. PRIMA is a computational tool that is available to model only the primary electron motions inside the discharge chamber. It does not include the other physics which are modeled in XOOPIC such as electric field effects, modeling of secondary electron, modeling of ions, etc. PRIMA gives two primary outputs as results: primary electron confinement length and primary electron relative number density. The results obtained from PRIMA for the Hiatt and Wilbur's model have good comparisons (refs. 8 and 32) with Hiatt and Wilbur's results for the primary electron utilization factor. 
The relative primary electron number density, $n_{p e}^{*}$, is determined using, (ref. 6)

$$
n_{p e}^{*}=\frac{n_{p e}}{n_{p e, \max }}
$$

where $n_{p e}$ is the primary electron density at some location and $n_{p e \text {,max }}$ is the maximum primary electron number density. The values of relative primary electron number density vary between 0 and 1 . Generally the value of 1 is observed at the cathode tip. The maximum value of primary electron number density observed at $300 \mathrm{~ns}$ is $3.3 \times 10^{18} \mathrm{\#} / \mathrm{m}^{3}$ from XOOPIC. This value can be used to convert PRIMA's relative primary electron number density results into an actual primary electron number density distributions. This converted result is shown in figure 16.

Comparing figure 16 with figure 10 we can see that the number density contours at PRIMA and XOOPIC follow the same basic trend toward the side wall magnet, but different towards the back wall magnet. More than likely this is due to the fact that XOOPIC results have not made it to steady state. In future work XOOPIC will be taken out to steady state and this comparison will be made again. At this time all that will be said is that it appears as if results from XOOPIC are beginning to look like those from PRIMA.

At this time XOOPIC is not capable of giving primary electron confinement length so a comparison for this output can not be made. In the future XOOPIC will be modified to produce this quantity.

\section{Future Work}

The results presented in this work are preliminary in the computational modeling of an ion engine discharge chamber. In this work it is demonstrated that using a computational tool called XOOPIC it is possible to model the primary electrons, secondary electrons and ions inside the discharge chamber including the electric and magnetic field effects. The results are studied on an experimental design of discharge chamber of an ion engine. In the future it is hoped to get the steady state results for this model. Currently XOOPIC is not running in a parallelized mode. Parallelization has to be utilized in modeling this discharge chamber design to reach steady state results.

In addition, the Debye length limit poses a big problem in resolving the computational mesh, thus non uniform mesh model needs to be explored.

\section{Conclusion}

In this work it is demonstrated that primary electrons, secondary electrons, and ions can be simultaneously tracked throughout a discharge chamber while the detailed electrical fields are being updated to include the effects of the charged particles. In this work a number of results are presented for a $9.2 \mathrm{~cm}$ diameter discharge chamber that is $11.8 \mathrm{~cm}$ long. In these it is seen that the ions move into the stronger magnetic field regions more than the electrons. The primary electrons tend to move out away from the cathode much quicker than the ions or secondary electrons. The primary electrons tend to run along magnetic field lines that run from the cathode to the anode. The secondary electrons tend to stay in regions where the ions are located. The electrical fields are greatly affected by the charges present at the cathode. In general the cathode region is the location of highest particle number density for all particles computed. While not shown in this paper a number of other results can be obtained from XOOPIC like the electric field, the average kinetic energy and the particle flux to the walls and to the anode surfaces. The computer program XOOPIC is capable of producing a great deal of information on the discharge present in an ion engine.

\section{References}

1. Verboncoeur, J.P., Langdon, A.B., and Gladd, N.T., “An object-oriented electromagnetic PIC code,” Comp. Phys. Comm., vol. 87, pp. 199-211, 1995. Download XOOPIC at http://ptsg.eecs.berkeley.edu/, 2005.

2. Tech-X Corporation, OOPIC user manual web site, http://www.techxhome.com/products/OOPIC_Pro/support/index.php, 2005.

3. Bruhwiler, D.L. et al., "Particle-in-cell simulations of plasma accelerators and electron-neutral collisions," Physical Review Special Topics-Accelerators and Beams, vol. 4, pp. 101302(1-13), 2001.

4. Luginsland, J.W., McGee, S., and Lau, Y.Y., "Virtual cathode formation due to electromagnetic transients," IEEE Transactions on Plasma Science, vol. 26, no. 3, pp. 901-904, 1998. 
5. Morris, D., and Gilchrist, B., "Electon emission for electric propulsion: reducing power by mitigating space charge limits," AIAA paper number 2004-3497, 2004.

6. Luginsland, J.W., Lau, Y.Y., and Gilgenbach, R.M., "Two-dimensional Child-Langmuir law," Physical Review Letters, vol. 77, no. 22, pp. 4668-4670, 1996.

7. Arakwa, Y., and Ishihara, K., "A numerical code for cusped ion thrusters," International Electric Propulsion Conference, IEPC-91-118, 1991.

8. Arakwa,Y., and Yamada, T., "Monte Carlo simulations of primary electron motions in cusped discharge chambers," International Electric Propulsion Conference, AIAA-90-2654, 1990.

9. Arakwa, Y. and Wilbur, P.J., "Finite element analysis of plasma flows in cusped discharge chambers," Journal of Propulsion and Power, vol.7, pp. 125-128, 1991.

10. Brophy, J.R. and Wilbur, P.J., "Simple performance model for ring and line cusp ion thruster," AIAA Journal, vol. 23, pp. 1731-1736, 1985.

11. Mahalingam, S., and Menart, J., "Primary electron modeling in the discharge chamber of an ion engine," AIAA paper number 2002-4262, 2002.

12. Hirakawa, M., and Arakawa, Y., "Plasma particle simulation in cusped ion thrusters," International Electrical Propulsion Conference, IEPC-93-242, 1993.

13. Wirz, R., and Katz, I., "2-D Discharge chamber model for ion thrusters," AIAA paper number 2004-4107, 2004.

14. Stueber, T.J., "Discharge chamber primary electron modeling activities in 3-dimensions," AIAA paper number 2004-4105, 2004.

15. Hiatt, J. and Wilbur, P., "Ring cusp discharge chamber performance optimization," International Electric Propulsion Conference, AIAA-85-2007, 1985.

16. Griffiths, D.J., Introduction to Electrodynamics, $2^{\text {nd }}$ Edition, Prentice Hall, Englewood Cliffs, New Jersey, (1989).

17. Birdsall, C.K., and Langdon, A.B., Plasma Physics via Computer Simulation, Adam Hilger, Bristol, 1991.

18. Domonkos, M.T., "A particle and energy balance model of the orificed hollow cathode," AIAA paper number 2002-4240, 2002.

19. Patterson, M.J. et al., "Plasma contactor development for space station," $23^{\text {rd }}$ International Electric Propulsion Conference, IEPC-93-246, 1993.

20. Vincenti, W.G., and Kruger, C.H., Introduction to Physical Gas Dynamics, Krieger Publishing Company, Malabar, Florida, (1965).

21. Birdsall, C.K., "Particle-in-cell charged-particle simulations, plus Monte-Carlo collisions with neutral atoms, PIC-MCC," IEEE Transactions on Plasma Science, vol. 18, no. 2, pp. 65-85, April 1991.

22. Peng, X., Keefer, D., and Ruyten, W., "Plasma particle simulation of electrostatic ion thrusters," $21^{\text {st }}$ International Electric Propulsion Conference, AIAA-90-2647, 1990.

23. Vahedi, V., and Surendra, M., "A Monte Carlo collision model for the particle-in-cell method: applications to argon and oxygen discharges," Comp. Phys. Comm., vol. 87, pp. 179-198, 1995.

24. Yee, K.S., "Numerical solution of initial boundary value problems involving Maxwell's equations in isotropic media," IEEE Trans. Antennas Propag., vol. 14, pp. 302-307, 1966.

25. Ansoft Corporation, Maxwell 2-D web site, http://www.ansoft.com/products/em/max2d/index.cfm, 2005.

26. Menart, J.A., "Magnetic Field Codes," NASA internal memo, August 13, 1998.

27. Surendra, M., Graves, D.B., and Jellum, G.M., "Self-consistent model of a direct-current glow discharge: treatment of fast electrons," Physical Review A, vol. 41, no. 2, pp. 1112-1125, 1990.

28. Rapp, D. and Englander-Golden, P., "Total cross sections for ionization and attachment in gases by electron impact. I. positive ionization," Journal of Chemical Physics, vol. 43, pp. 1464-1479, 1965.

29. Hockney, R.W., and Eastwood, J.W., Computer Simulation Using Particles, Adam Hilger, Bristol, 1989.

30. Van Noord, J.L., "Thermal model of an ion thruster," Ph.D. Dissertation, University of Michigan, 1999.

31. Foster, J.E., and Patterson, M.J., "Plasma emission characteristics from a high current hollow cathode in an ion thruster discharge chamber," AIAA paper number 2002-4102, 2002.

32. Shirin, D.S., Mahalingam, S., and Menart, J.A., "Computational study of primary electrons in the cusp region of an ion engine's discharge chamber," AIAA paper number 2004-4109, 2004. 


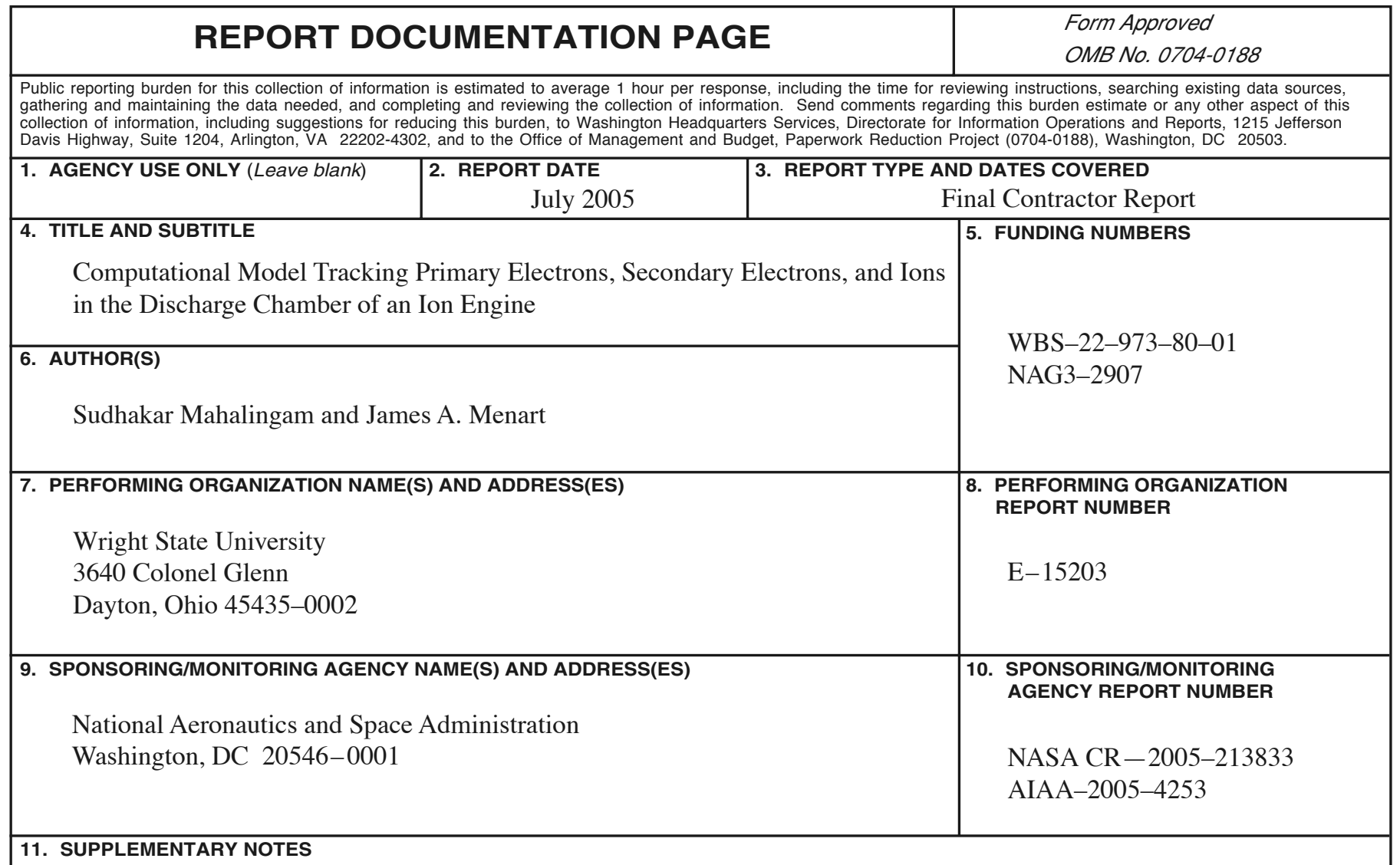

Prepared for the 41st Joint Propulsion Conference and Exhibit cosponsored by the AIAA, ASME, SAE, and ASEE, Tucson, Arizona, July 10-13, 2005. Project Manager, Thomas J. Stueber, Power and Electrical Propulsion Division, NASA Glenn Research Center, organization code RPP, 216-433-2218.

\begin{tabular}{|l|l|}
\hline 12a. DISTRIBUTION/AVAILABILITY STATEMENT & 12b. DISTRIBUTION CODE
\end{tabular}

Unclassified - Unlimited

Subject Category: 75

Available electronically at http://gltrs.grc.nasa.gov

This publication is available from the NASA Center for AeroSpace Information, 301-621-0390.

13. ABSTRACT (Maximum 200 words)

Computational modeling of the plasma located in the discharge chamber of an ion engine is an important activity so that the development and design of the next generation of ion engines may be enhanced. In this work a computational tool called XOOPIC is used to model the primary electrons, secondary electrons, and ions inside the discharge chamber. The details of this computational tool are discussed in this paper. Preliminary results from XOOPIC are presented. The results presented include particle number density distributions for the primary electrons, the secondary electrons, and the ions. In addition the total number of a particular particle in the discharge chamber as a function of time, electric potential maps and magnetic field maps are presented. A primary electron number density plot from PRIMA is given in this paper so that the results of XOOPIC can be compared to it. PRIMA is a computer code that the present investigators have used in much of their previous work that provides results that compare well to experimental results. PRIMA only models the primary electrons in the discharge chamber. Modeling ions and secondary electrons, as well as the primary electrons, will greatly increase our ability to predict different characteristics of the plasma discharge used in an ion engine.

14. SUBJECT TERMS

Electric propulsion thruster; Discharge chamber; Simulation

\begin{tabular}{|c|c|c|}
\hline $\begin{array}{c}\text { 17. SECURITY CLASSIFICATION } \\
\text { OF REPORT } \\
\text { Unclassified }\end{array}$ & $\begin{array}{c}\text { 18. SECURITY CLASSIFICATION } \\
\text { OF THIS PAGE } \\
\text { Unclassified }\end{array}$ & $\begin{array}{c}\text { 19. SECURITY CLASSIFICATION } \\
\text { OF ABSTRACT } \\
\text { Unclassified }\end{array}$ \\
\hline
\end{tabular}

NSN 7540-01-280-5500

Standard Form 298 (Rev. 2-89)

Prescribed by ANSI Std. Z39-18 298-102 

